\title{
Tandem Repeat Variation
}

National Cancer Institute

\section{Source}

National Cancer Institute. Tandem Repeat Variation. NCI Thesaurus. Code C45680.

An inherited polymorphism in eukaryotic DNA that results from variations in the number of tandem repeat nucleotide sequences, which are end-to-end adjacent copies of a short (2 to 60 nucleotides) DNA sequence located between two restriction endonuclease recognition sites. These are referred to as variable number of tandem repeats (VNTR), and the number of alleles at these loci can vary from 2 to more than 20 . VNT Rs provide important genetic information that is used to identify an individual via the DNA fingerprinting technique. 